zentraler Karrierebestandteil von Ministern zu sein. Insgesamt kann jedoch auch hier der Trend zu vielfältigeren Wegen in das Ministeramt beobachtet werden, auch wenn die kulturellen, historischen und politischen Rahmenbedingungen in den einzelnen Staaten diesen Trend verschiedentlich brechen, abschwächen oder verstärken.

Vor diesem Hintergrund regte der konzeptionelle Beitrag von Luca Verzichelli (Universität Siena) eine bessere Integration der verschiedenen Datengrundlagen und Forschungsstränge an. Darin wurden die theoretischen und empirisch-praktischen Herausforderungen für die weitere Forschung angesprochen, die sich aufgrund der in den einzelnen Beiträgen geschilderten Entwicklungen ergeben. Die skizzierten Forschungsergebnisse deuten demnach auf strukturelle Veränderungen in den Karriereverläufen europäischer Minister hin. Die bisher fokussierten Karriereprozesse - Rekrutierung, Zirkulation und generelle Verlaufsmuster von Karrieren - werden, so Verzichelli, von diesen Veränderungen so sehr beeinflusst, dass es eines erweiterten Analyserahmens zur Untersuchung von Ministerkarrieren bedarf. Als potenzielle Einflussgrößen benannte er den Bedeutungsgewinn von Kernressorts, der eine größere Unabhängigkeit der jeweiligen Minister von ihrer Partei zur Folge hat, sowie die Europäisierung vieler Politikfelder. Sein vorgeschlagener Forschungsansatz verspricht gegenüber den herkömmlichen Perspektiven ein größeres Erklärungspotential für die Varianz von Ministerkarrieren.

Die in Dornburg diskutierten Befunde zeigen, dass das eingangs genannte Diktum von Bagehot durch verschiedene Entwicklungen herausgefordert wird. Insofern stellte die Tagung einen ersten Schritt dar, die Erforschung der personellen Verknüpfung zwischen Parlament und Regierung konzeptionell neu auszurichten. Eine diesen Ansatz weiterverfolgende Forschung verspricht zugleich Erkenntnisse über das generelle Verhältnis der beiden Verfassungsinstitutionen und damit zur Dynamik des parlamentarischen Regierungssystems.

Sebastian Dörl und André Nagel

\title{
Die Gesellschaft zum Studium strukturpolitischer Fragen (Strukturgesellschaft). Veranstaltung zum 50-jährigen Bestehen am 5. März 2009
}

Am 5. März 2009 feierte die „Gesellschaft zum Studium strukturpolitischer Fragen“ (kurz: Strukturgesellschaft) in den Räumen der Deutschen Parlamentarischen Gesellschaft ihr 50-jähriges Bestehen. Festredner waren der Präsident der Parlamentarischen Gesellschaft Prof. Dr. Heinz Riesenhuber und der Träger des Friedenspreises des deutschen Buchhandels von 2007 Prof. Dr. Wolf Lepenies. Er sprach zu dem Thema „Provinzielles Denken und Weltbürgertum: die amerikanisch-europäischen Beziehungen in der Ära Obama“.

Der Präsident der Strukturgesellschaft Dr. Ludolf von Wartenberg wies in seiner Laudatio darauf hin, dass die Gesellschaft in der breiten Öffentlichkeit wenig bekannt, aber bei aller Zurückhaltung im Auftreten eine im Parlament weitgehend anerkannte Institution sei, die Hilfe im politischen Entscheidungsprozess leisten könne, insbesondere in der Wirtschafts-, Finanz-, Sozial- und Gesellschaftspolitik. Die Strukturgesellschaft nimmt unter den Institutionen im Umfeld des Bundestages eine besondere und einmalige Stellung ein. Hinter dem etwas umständlichen Namen verbirgt sich die älteste und bekannteste dieser Vereini- 
gungen. Außerdem zeichnet sie sich durch eine besondere Nähe zum Parlament aus. Sie bildet eine überparteiliche Schnittstelle zwischen Politik, Verwaltung, Wirtschaft und Wissenschaft.

Sie wurde im Februar des Jahres 1959 gegründet. Die Zielsetzung der Gesellschaft wurde folgendermaßen beschrieben: „Ihre Aufgabe sollte es sein, auf den Einzelgebieten der Steuergesetzgebung, des Wirtschafts- und Gesellschaftsrechts, der Sozialgesetzgebung, der Verkehrspolitik und der Soziologie wissenschaftliche Untersuchungen unter dem Blickwinkel strukturpolitischer Auswirkungen anzustellen. " ̈̈hnlich ist in der Präambel der Satzung der Gesellschaft von 1959 festgehalten: „Diese Veränderungen [der Wirtschaftsstrukturen] bedürfen einer laufenden wissenschaftlichen Beobachtung und Analyse, um sachgerechte Entscheidungen bei Gesetzgebung, Politik und Verwaltung zu unterstützen.“

Damals ging die Aufbauphase der Bundesrepublik nach dem Kriege zu Ende und der Politik wurde mehr und mehr bewusst, dass auch der Strukturwandel der Wirtschaft einer politischen Gestaltung bedurfte. „Die wirtschaftlichen und gesellschaftlichen Strukturen unseres Volkes sind einem ständigen Wandel unterworfen. Zwei Kriege mit Sachwertvernichtungen in bisher ungekanntem Ausmaß, zwei Währungsschnitte mit folgenreichen Eingriffen in die Eigentumsverteilung, eine außerordentliche Umschichtung in Produktion und Absatz in Folge der technischen Neuerungen und das Zusammenwachsen in übernationale Wirtschaftseinheiten haben die überkommenen Formen der Gesellschafts- und Wirtschaftsstruktur grundlegend verändert und werden auch zukünftig wesentliche Veränderungen hervorrufen." Ein besonders wichtiger Aspekt war damals, dass manche Institutionen, die eine beratende Funktion für die Politik ausüben, noch nicht eingerichtet waren. So wurde der „Sachverständigenrat zur Begutachtung der gesamtwirtschaftlichen Entwicklung“ erst 1963 von Ludwig Erhard gesetzlich eingeführt. Aus der Präambel der Satzung geht weiter hervor, dass es Aufgabe der Gesellschaft ist, „Wissenschaft, wirtschaftliche und gesellschaftliche Praxis, Gesetzgebung und Verwaltung zusammenzuführen. Sie bedient sich dazu in erster Linie ihrer Beiräte. Die wissenschaftliche Untersuchung der wirtschafts- und gesellschaftspolitischen Strukturprobleme erstreckt sich sowohl auf umfassende strukturpolitische Grundsatzfragen als auch auf viele Einzelgebiete. Sie geht vom Ordnungsrahmen der Sozialen Marktwirtschaft aus“. Die zentrale Rolle in der Strukturgesellschaft kommt also den Beiräten zu. In ihnen erfolgt die eigentliche Arbeit.

Heute verfügt die Gesellschaft über 13 Beiräte, die weitgehend die wirtschaftsrelevanten Ausschüsse des Bundestages abbilden. Die Zahl und die Zusammensetzung wechseln je nach politischem Bedarf. So gibt es derzeit keinen Beirat Agrarwirtschaft. Die heute existierenden Beiräte sind nach Alphabet geordnet: Arbeit und Soziales; Bildung und Forschung; Energie; Europa; Finanzen, Steuern, Geld und Kredit; Gesundheit; Handel und Verbraucherschutz; Medien, Internet und Telekommunikation; Sicherheit und Verteidigung; Umwelt, Naturschutz und Reaktorsicherheit; Verkehr, Bau und Stadtentwicklung; Welthandel; Wirtschaft und Technologie.

Die Beiratssitzungen laufen in der Regel nach folgendem Muster ab: Der Vorsitzende eines Beirates ist stets ein Abgeordneter. Dabei gibt es in aller Regel keine Probleme, Parlamentarier für dieses Amt zu gewinnen, denn die anfallende Arbeit bewegt sich im engen Rahmen und bietet ihnen die Gelegenheit, mit ihrer „Fachgemeinde“ in Berlin und darüber hinaus in Kontakt zu treten. Besonders für junge Abgeordnete ist das attraktiv.

„Parlamentsnahe Themen“werden in den Sitzungen behandelt. Das heißt, es sind solche Gegenstände, die derzeit auf der Tagesordnung des Bundestages stehen oder von denen 
man annehmen kann, dass sie demnächst dorthin kommen. Dabei werden heute nicht allein strukturelle Fragen der Wirtschaft, sondern auch Konjunktur-, Ordnungs- und Rechtsfragen erörtert, wenn sie nur einen wirtschaftlichen Bezug haben.

Die Sitzungen beginnen mit Kurzstatements von in der Regel drei Vortragenden. Es sind vertreten: die Wissenschaft, sie nimmt in aller Regel die Einordnung des zu behandelnden Sachverhalts in das wissenschaftliche Ideengebäude vor; die Bundesverwaltung, die für die Umsetzung des politisch Gewollten verantwortlich ist und ein Betroffener (Unternehmens- oder Verbandsvertreter), der die Auswirkungen einer Maßnahme in der Praxis zu tragen hat. Die Politik wird durch den Vorsitzenden vertreten. Die Referenten sind meist hervorgehobene Vertreter ihrer Profession wie Vorstandsvorsitzende, Geschäftsführer, Professoren, Staatssekretäre, Abteilungsleiter oder Gewerkschaftsvertreter.

So wird ein breites Spektrum von Meinungen abgedeckt, und es wird darauf geachtet, dass unterschiedliche und kontroverse Positionen zu Wort kommen. Dieses Verfahren sichert eine hohe Informationsdichte, eine objektive Durchleuchtung des Sachverhalts und das Finden oder Sublimieren von Lösungen. Ein verbesserter Informationsstand aller Teilnehmer wird auf diesem Wege ebenso erreicht wie das Interesse an gemeinsamer Arbeit an der gestellten Aufgabe.

Danach folgen Fragen aus dem Publikum an das Podium. Eigenständige Beiträge sollen nur im kürzesten Rahmen erfolgen. Unterschiedliche Positionen werden im Disput herausgearbeitet. Als Ergebnisse der Sitzungen sind festzuhalten: Sachverhalte werden aufgeklärt, unterschiedliche Auffassungen treffen aufeinander, Abgeordnete nehmen Informationen auf und moderieren auch, Forderungen an die Politik werden gestellt.

Die einzelnen Themen ergeben sich sehr häufig aus der tagespolitischen Diskussion. Sie werden auch in einer gemeinsamen Abrede zwischen den Mitgliedern, den Beiratsvorsitzenden sowie dem Wissenschaftlichen Beirat der Gesellschaft ausgesucht.

Dieses Konzept wird sehr flexibel gehandhabt. Bei einer anderen Variante der Sitzungen, die sich in letzter Zeit herausgebildet hat, trägt ein Wissenschaftler, der ein eigenständiges Konzept zu einem Problem erarbeitet hat, dieses vor und stellt es den Fachvertretern und der Politik zur Diskussion.

Die Einladungen zu den Sitzungen gehen über den Kreis der Mitglieder hinaus, zum Beispiel an alle Abgeordneten des Bundestages. Darüber hinaus wird ein breites Spektrum von Personen angesprochen, die sich beruflich mit dem anstehenden Thema befassen. Allerdings wird keine Presse zugelassen, da sich sonst der Charakter der Diskussionen verändern würde. Es würde insbesondere weniger offen gesprochen und verstärkt Verbands- und Parteistandpunkte vertreten. Aus dem gleichen Grund werden keine Protokolle angefertigt. Charts werden zur Verfügung gestellt, sofern es die Autoren zulassen.

Heute haben die Sitzungen im Schnitt 50 Teilnehmer, wobei auch Veranstaltungen mit mehr als 100 vorkommen. Die Sitzungen finden in den Räumen des Bundestages in dessen Mittagspause statt. Es wird pünktlich um 13.00 Uhr begonnen und ebenso pünktlich um 15.00 Uhr geendet. Durch diese Terminierung sind insbesondere Abgeordnete in der Lage, an den Sitzungen teilzunehmen. Neben Abgeordneten finden sich unter den Teilnehmern die letzte Arbeitsebene unter der Entscheidungsebene in Unternehmen und Verbänden, deren Repräsentanten, Mitarbeiter von Abgeordneten, Repräsentanten von Landesvertretungen, Vertreter ausländischer Botschaften, Professoren, wissenschaftliche Mitarbeiter von Hochschulen usw. - also typischerweise der Personenkreis, den man als Multiplikatoren bezeichnet. 
Das Besondere der Strukturgesellschaft liegt darin, dass sie eine starke Nähe zum Bundestag aufweist: sie ist eine Gründung aus dem Parlament; viele ihrer Mitglieder sind Parlamentarier; die Vorsitzenden der Beiräte sind allesamt Abgeordnete; Parlamentarier nehmen an den Sitzungen teil; Diskussionen in den Beiräten werden in den Kreis der Abgeordneten getragen. Besonders wichtig ist, dass die diskutierten Themen sich an der Tagesordnung des Parlaments orientieren und mit Parlamentariern abgesprochen werden. Die Parlamentsnähe ergibt sich außerdem und besonders aus der Themenstellung.

Man könnte der Gesellschaft vorhalten - und dies ist auch geschehen -, sie sei eine besonders effektive Art des Lobbyings der Wirtschaft im Parlamentsbereich. Dies ist ein Fehlurteil. Lobbying versucht, Interessen Einzelner gegenüber dem Gesetzgeber zu verdeutlichen und durchzusetzen. Bei der Strukturgesellschaft handelt es sich um eine offene, neutrale Plattform, auf der jeder seine Position darlegen kann. Verbände wollen Einfluss ausüben. Das können sie aber nur sehr bedingt in einer offenen Diskussion, weil ihre Argumente sofort in Frage gestellt werden können. Außerdem ist die Mitgliedschaft der Gesellschaft so heterogen, dass eine Ausrichtung auf ein einziges zu verfolgendes Ziel unmöglich ist.

Es stellt sich daher die Frage, wo der Mehrwert einer Teilnahme an den Beiräten und in einer Mitgliedschaft liegt. Ein erster Grund mag darin liegen, dass hier Abgeordnete als Ansprechpartner zur Verfügung stehen. Anhörungen im Bundestag sind heute häufig steril. Sie finden in der Öffentlichkeit statt, und es werden die bekannten Standpunkte ausgetauscht. Auch diejenigen, die nicht zu Anhörungen eingeladen werden, kommen in den Beiratssitzungen zu Wort. Es wird zwischen dem Podium und dem „Plenum“ diskutiert. Man kann die eigenen Argumente prüfen, man kann sich aber auch nur schnell, sachkundig und umfassend über ein wirtschaftspolitisches Thema informieren.

Wenn die Strukturgesellschaft einen Anspruch hat, besteht er in der Verpflichtung auf die Soziale Marktwirtschaft. Sie stellt marktwirtschaftliche Lösungen ins Zentrum ihrer Arbeit und präsentiert sie einem breiten Publikum zur Diskussion. Insofern ist sie ein Gegengewicht zu dem heute in allen Parteien und Fraktionen weit verbreiteten wirtschaftspolitischen Interventionismus, der den politischen Eingriff in den Wirtschaftsprozess als grundsätzlich zulässig und notwendig erachtet. So wirkt die Gesellschaft über ihre Parlamentsnähe in den Bundestag hinein. Darüber hinaus geht es um das Bereitstellen von politischem, wirtschaftlichem und technischem Wissen und Sachverstand.

Berichtet wird, dass in den 50er Jahren regelmäßig Mittagessen von wirtschaftspolitischen Entscheidungsträgern der CDU/CSU-Bundestagsfraktion stattfanden, um die politische Linie zu wichtigen wirtschaftspolitischen Fragen festzulegen. Aus diesen Treffen ist offenbar die Strukturgesellschaft entstanden. Vorarbeiten zur Schaffung der Gesellschaft, die sich den oben formulierten Zielen annehmen sollte, sind seit Oktober 1958 nachweisbar. Die Initiative ging von Kurt Schmücker aus, der damals Stellvertretender Fraktionsvorsitzender und Vorsitzender des Arbeitskreises Wirtschaft der CDU/CSU war und später Nachfolger von Ludwig Erhard als Bundeswirtschaftsminister wurde. Mitinitiator war der damalige Postminister und spätere langjährige Bundestagspräsident Richard Stücklen.

Die Strukturgesellschaft war also zunächst eine reine CDU/CSU-Veranstaltung. Ihre Gründer waren, nach den Lebensläufen der Beteiligten zu urteilen, keineswegs Hinterbänkler ihrer Fraktion. Sie hatten einen starken Bezug zum unternehmerischen Mittelstand.

Die Gesellschaft nahm nach der Gründung einen raschen Aufstieg. Ende 1959 hatte sie lediglich 37 Mitglieder, 1968 waren es mit 164 schon fast fünfmal so viele. Die Gesellschaft 
war laut Satzung offen für jede natürliche und juristische Person. Der Kontakt zur FDP war fast von Beginn an immer gegeben. Der Kontakt von Schmücker, dem ersten Vorsitzenden, zur SPD war ebenfalls gut. Zum SPD-Mittelstandsvertreter Erwin Lange (Essen) pflegte er freundschaftliche Beziehungen, vermutlich weil auch er Buchdrucker von Beruf war. Lange soll zu den Sitzungen gekommen sein.

Der Umzug der Gesellschaft von Bonn nach Berlin erfolgte am 1. Oktober 1999, um auch nach dem Umzug des Bundestages die Parlamentsnähe beizubehalten. Damit kam es zu einer Umstrukturierung der Mitgliedschaft. Viele Mitglieder, die nicht von Bonn nach Berlin wechselten, traten aus. Gleichzeitig wurden in Berlin neue Mitglieder gefunden, und inzwischen hat sich die Mitgliederzahl wieder stabilisiert, auf heute 247, darunter 179 natürliche Personen (davon 59 Bundestagsabgeordnete), 47 Verbände und 21 Unternehmen.

Mit dem Umzug nach Berlin wurde auch die Öffnung der Gesellschaft für andere Parteien mit Nachdruck betrieben. Dies war der ausdrückliche Wunsch der Mitglieder. Die anderen der Marktwirtschaft verpflichteten Fraktionen wachsen seither langsam in einen Prozess der Integration in die Gesellschaft hinein. Derzeit werden mit zwei Ausnahmen alle Stellvertreter der Beiratsvorsitzenden von der FDP gestellt. Dieser Partei wurde damit entsprechend ihrer Funktion im Parlament ein „Oppositionsbonus“ gegeben. Die SPD ist im Vorstand und im Beirat Europa, Bündnis 90/Die Grünen sind seit neuerer Zeit im Vorstand vertreten.

Bemerkenswert ist, dass die Gesellschaft in den 50 Jahren ihres Bestehens lediglich drei Vorstandsvorsitzende gehabt hat. 1963 wurde Erhard Bundeskanzler und Schmücker sein Nachfolger als Bundeswirtschaftsminister. Den Vorsitz der Gesellschaft gab Schmücker damit nach vier Jahren ab. Neuer Vorsitzender wurde Dr. Ernst Müller-Hermann. Nach 21 Jahren legte er sein Amt aus Altersgründen im Jahre 1985 nieder. Ihm folgte am 28. November 1985 von Wartenberg, der bis heute die Gesellschaft führt. Er hat das Amt aus seiner Abgeordnetentätigkeit in andere berufliche Funktionen mitgenommen: Parlamentarischer Staatssekretär im Bundesministerium für Wirtschaft, danach Hauptgeschäftsführer des Bundesverbandes der Deutschen Industrie.

Die Gesellschaft hat in den 50 Jahren ihres Bestehens verschiedene Phasen durchlaufen und Wandlungen erfahren. Die wichtigste ist die Gewinnung von Personen aus allen Bundestagsfraktionen, die für eine der Freiheit verpflichtete Wirtschafts- und Gesellschaftsordnung eintreten. Die Arbeit der Gesellschaft orientiert sich nicht an theoretischen Fragen, sondern bemüht sich vielmehr um das handfeste Bedürfnis, Wissenschaft, wirtschaftliche und gesellschaftliche Praxis, Gesetzgebung und Verwaltung zu konkreten Sachfragen der Gesetzgebung und zu Themen von allgemeinem Interesse zusammenzuführen. Für viele Abgeordnete des Bundestages sind die Sitzungen der Beiräte der Strukturgesellschaft deshalb eine wertvolle Entscheidungshilfe. Die Gesellschaft ist konzeptionell gut aufgestellt und im Parlament und der interessierten Öffentlichkeit anerkannt. Dies ist eine gute Voraussetzung für ihre Arbeit in der Zukunft. 